\title{
PRESSURE DISTRIBUTION IN A POROUS SQUEEZE FILM BEARING LUBRICATED WITH A HERSCHEL-BULKLEY FLUID
}

\author{
A. WALICKA* and P. JURCZAK \\ University of Zielona Góra \\ Faculty of Mechanical Engineering \\ ul. Szafrana 2, P65-516 Zielona Góra, POLAND \\ E-mail: A.Walicka@ijame.uz.zgora.pl \\ P.Jurczak@ibem.uz.zgora.pl
}

\begin{abstract}
The influence of a wall porosity on the pressure distribution in a curvilinear squeeze film bearing lubricated with a lubricant being a viscoplastic fluid of a Herschel-Bulkley type is considered. After general considerations on the flow of the viscoplastic fluid (lubricant) in a bearing clearance and in a porous layer the modified Reynolds equation for the curvilinear squeeze film bearing with a Herschel-Bulkley lubricant is given. The solution of this equation is obtained by a method of successive approximation. As a result one obtains a formula expressing the pressure distribution. The example of squeeze films in a step bearing (modeled by two parallel disks) is discussed in detail.
\end{abstract}

Key words: Herschel-Bulkley lubricant, porous bearing, squeeze film, thrust bearing.

\section{Introduction}

Steady state radial flows and time-dependent squeezing flows of yield-viscoplastic fluids are encountered in a variety of fields [1-7]. These flows are found in fabrication operations such as stamping, injection molding, and sheet forming. Also, material properties of highly viscous fluids are measured with a device called the "plastometer" which incorporates a parallel-disk squeeze flow geometry [2, 8, 9]. In addition, such flows are encountered in lubrication systems, and there is a considerable interest as to the degree which viscoplastic additives enhance the load-bearing capacity of the lubricant.

The flows of Newtonian fluids in the clearance of a thrust bearing with impermeable surfaces have been examined theoretically. The bearing walls have been modelled as two disks, two conical or spherical surfaces. A more general case is established by the bearing formed by two surfaces of revolution [10].

Porous bearings have been widely used in industry for a long time [11-15]. Basing on the Darcy model Morgan and Cameron first presented theoretical research on these bearings [13].

Recently the problem of slide bearings with porous walls lubricated with a Bingham fluid was taken up by Walicki et al. [16] but the problem of a porous squeeze film bearing lubricated with the same lubricant was presented by Walicka [17].

From various models of fluids with a yield shear stress the Herschel-Bulkley fluid flow frequently appears in many industrial branches: polymer processing, food processing, metal processing, etc. It also appears in tribology modelling of semi-fluid lubricants flow $[18,19]$.

The purpose of this study is to investigate the pressure distribution in a clearance of a squeeze film bearing formed by two surfaces of revolution, having a common axis of symmetry, as shown in Fig.1; the lower one of these surfaces is connected with a porous layer. The analysis is based on the assumption that the porous matrix consists of a system of capillaries of very small radii restricting the lubricant flow through the matrix in only one direction.

\footnotetext{
* To whom correspondence should be addressed
} 
The lubricant is assumed to be a Herschel-Bulkley type for which the constitutive equation has the following form $[20,21]$

$$
\tau=\tau_{0}+(\mu \dot{\gamma})^{\frac{1}{m}}
$$

where $\tau$ is the shear stress, $\tau_{0}$ is the yield shear stress, $\mu$ is the coefficient of plastic viscosity, $\dot{\gamma}$ is the rate of deformation, $m$ is the exponent in a Herschel-Bulkley fluid.

\section{Analysis of the Herschel-Bulkley fluid flow in a bearing clearance}

The flow configuration is shown in Fig.1. The upper boundary of a porous layer is described by function $\mathrm{R}(x)$, which denotes the radius of this boundary. The fluid film thickness is given by the function $h(x, t)$. An intrinsic curvilinear orthogonal co-ordinate system $(x, \vartheta, y)$ is also depicted in Fig.1.
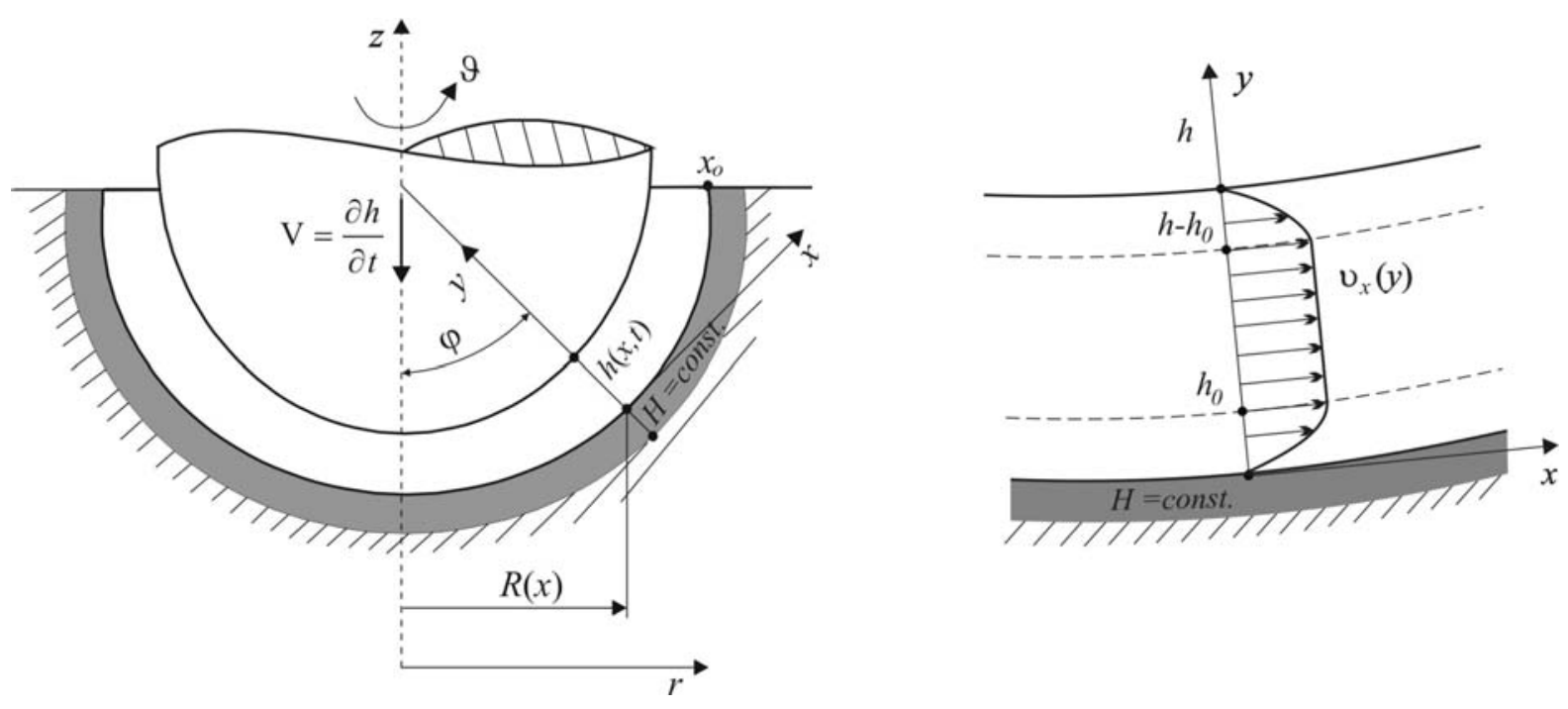

Fig.1. Configuration of a thrust bearing with one porous wall.

By using the assumptions typical for the flows in a thin layer the equations of motion for a HerschelBulkley fluid for axial symmetry can be presented in the form [22-24]

$$
\begin{aligned}
& \frac{1}{R} \frac{\partial\left(R v_{x}\right)}{\partial x}+\frac{\partial v_{y}}{\partial y}=0 \\
& \frac{\partial p}{\partial x}=\frac{\partial \Lambda_{y x}}{\partial y} \\
& \frac{\partial p}{\partial y}=0
\end{aligned}
$$

where the non-zero component of the stress tensor is $[19,23,24]$ 


$$
\Lambda_{y x}= \begin{cases}\tau_{0}+\left(\mu \frac{\partial v_{x}}{\partial y}\right)^{\frac{1}{m}} & \text { for } \\ -\tau_{0}+\left(-\mu \frac{\partial v_{x}}{\partial y}\right)^{\frac{1}{m}} & y \geq h-h_{0},\end{cases}
$$

In the flow of a fluid with yield shear stress there exists a quasi-solid core bounded by surfaces lying at

$$
y=h_{0} \quad \text { or } \quad y=h-h_{0} \quad \text { for which the shear stress is: }\left|\Lambda_{y x}\right|=\tau_{0} .
$$

The problem statement is complete after specification of boundary conditions which are

$$
\begin{array}{ll}
v_{x}(x, 0, t)=0, & v_{x}(x, h, t)=0, \\
v_{y}(x, 0, t)=V, & v_{y}(x, h, t)=\frac{\partial h}{\partial t}, \\
\left.\frac{\partial p}{\partial y}\right|_{x=0}=0, & p\left(x_{o}\right)=p_{o}
\end{array}
$$

here $V$ is the lubricant velocity on the upper boundary of a porous matrix, $p_{o}$ is the outlet pressure.

Putting Eqs (2.4) into Eq.(2.2) and integrating the resultant equations with respect to $y$ in the interval $0 \leq y \leq h$ and determining arbitrary constants from the boundary conditions (2.5) and (2.6) we obtain [23-25]

- for shear flow $\left(y \leq h_{0}\right.$ or $\left.y \geq h-h_{0}\right)$

$$
\mathrm{v}_{x s}=\frac{1}{\mu(m+1)}\left(-\frac{\partial p}{\partial x}\right)^{m}\left[h_{0}^{m+1}-\left(h_{0}-y\right)^{m+1}\right]
$$

for $y \leq h_{0}$ and

$$
\mathrm{v}_{x s}=\frac{1}{\mu(m+1)}\left(-\frac{\partial p}{\partial x}\right)^{m}\left\{h_{0}^{m+1}-\left[y-\left(h-h_{0}\right)\right]^{m+1}\right\}
$$

for $y \geq h-h_{0}$;

- for core flow $\left(h_{0} \leq y \leq h-h_{0}\right)$

$$
\mathrm{v}_{x c}=\frac{1}{\mu(m+1)}\left(-\frac{\partial p}{\partial x}\right)^{m} h_{0}^{m+1}
$$


The flow rate across the clearance is given by

$$
Q_{c}=h \hat{v}_{x}=2 \int_{0}^{h_{0}} v_{x s} d y+2 \int_{h_{0}}^{h / 2} v_{x c} d y=2 \int_{0}^{h_{0}} v_{x s} d y+v_{x c}\left(h-2 h_{0}\right),
$$

where $\hat{v}_{x}$ is the mean velocity in the clearance; after calculations one has

$$
h \hat{\mathrm{v}}_{x}=\frac{h^{m+2}}{12 \mu}\left(-\frac{\partial p}{\partial x}\right)^{m} \Phi^{(m)}(\chi)
$$

Here

$$
\chi=\frac{\tau_{0}}{\tau_{w}}, \quad h_{0}=\frac{\tau_{w}-\tau_{0}}{\left(-\frac{\partial p}{\partial x}\right)}=\frac{h}{2}(1-\chi), \quad \tau_{w}=\frac{h}{2}\left(-\frac{\partial p}{\partial x}\right), \quad \tau_{0}=\frac{1}{2}\left(h-2 h_{0}\right)\left(-\frac{\partial p}{\partial x}\right)
$$

$\tau_{w}$ - is the shear stress on the clearance wall.

The function $\Phi^{(m)}(\chi)$ is given as follows

$$
\Phi^{(m)}(\chi)=\frac{3}{2^{m-1}(m+2)}(1-\chi)^{m+1}\left(1+\frac{1}{m+1} \chi\right)
$$

\section{Modified Reynolds equation}

Integrating the equation of continuity (2.1) with respect to $y$ and determining the arbitrary constants from the boundary conditions $(2.7)$ we obtain $[19,24]$

$$
\frac{1}{R} \frac{\partial\left(R h \hat{\mathrm{v}}_{x}\right)}{\partial x}=-\frac{\partial h}{\partial t}+V
$$

or

$$
\frac{1}{R} \frac{\partial}{\partial x}\left[R h^{m+2}\left(-\frac{\partial p}{\partial x}\right)^{m} \Phi^{(m)}(\chi)\right]=-12 \mu\left(\frac{\partial h}{\partial t}-V\right)
$$

the modified Reynolds equation.

Considering the porous matrix as a system of capillaries (each of radius $r_{c}$ ) we may assume that the velocity components for the Herschel-Bulkley fluid flow in this matrix are given as [26]

where

$$
\overline{\mathrm{v}}_{x}=\Phi_{p}\left(-\frac{\partial \bar{p}}{\partial x}\right)^{m} \Psi^{(m)}(\Upsilon), \quad \overline{\mathrm{v}}_{y}=\Phi_{p}\left(-\frac{\partial \bar{p}}{\partial y}\right)^{m} \Psi^{(m)}(\Upsilon)
$$




$$
\begin{aligned}
& \Psi^{(m)}(\Upsilon)=\frac{(1-\Upsilon)^{m+1}}{m+3}\left[1+\frac{2 \Upsilon}{m+2}+\frac{2 \Upsilon^{2}}{(m+1)(m+2)}\right], \\
& \Upsilon=\frac{\tau_{0}}{\tau_{w}}=\frac{2 \tau_{0}}{r_{c}\left(-\frac{\partial p}{\partial x}\right)}, \quad \Phi_{p}=\frac{r_{c}^{m+1} \varphi_{p}}{2^{m} \mu}
\end{aligned}
$$

$\varphi_{p}$ - is the coefficient of porosity of the porous matrix.

Since the cross velocity component must be continuous at the porous wall-fluid film interface, one obtains from Eqs (3.1) and (3.2) 2 the modified Reynolds equation for the pressure distribution in the bearing with a porous wall

$$
\frac{1}{R} \frac{\partial}{\partial x}\left[R h^{m+2}\left(-\frac{\partial p}{\partial x}\right)^{m} \Phi^{(m)}(\chi)\right]=-12 \mu\left[\frac{\partial h}{\partial t}-\left.\Psi^{(m)}(\Upsilon) \Phi_{p}\left(-\frac{\partial \bar{p}}{\partial y}\right)^{m}\right|_{y=0}\right]
$$

To determine the last term in the brackets on the right hand side of Eq.(3.4) let us consider the lubricant flow in a porous matrix. The equation of continuity for the flow in the porous region has the same form as Eq.(2.1). By substituting Eqs (3.2) into Eq.(2.1) one obtains the following equation for pressure distribution in the porous region

$$
\frac{1}{R} \frac{\partial}{\partial x}\left[R \Psi^{(m)}(\Upsilon) \Phi_{p}\left(-\frac{\partial \bar{p}}{\partial x}\right)^{m}\right]+\frac{\partial}{\partial y}\left[\Psi^{(m)}(\Upsilon) \Phi_{p}\left(-\frac{\partial \bar{p}}{\partial y}\right)^{m}\right]=0 .
$$

Integrating this equation with respect to $y$ across the porous layer and using the Morgan-Cameron approximation we have [13]

$$
\left.\Psi^{(m)}(\Upsilon) \Phi_{p}\left(-\frac{\partial \bar{p}}{\partial y}\right)^{m}\right|_{y=0}=-\frac{H}{R} \frac{\partial}{\partial x}\left[R \Psi^{(m)}(\Upsilon) \Phi_{p}\left(-\frac{\partial \bar{p}}{\partial x}\right)^{m}\right]
$$

Note that on the porous wall-fluid film interface we have also

$$
\frac{\partial \bar{p}}{\partial x}=\frac{\partial p}{\partial x} \quad \text { and } \quad \Upsilon=\chi \frac{h}{r_{c}}
$$

When Eqs (3.6) and (3.7) are substituted into Eq.(3.4) the modified Reynolds equation takes the form

$$
\frac{1}{R} \frac{\partial}{\partial x}\left\{R\left[h^{m+2} \Phi^{(m)}(\chi)+12 \mu H \Phi_{p} \Psi^{(m)}(\Upsilon)\right]\left(-\frac{\partial p}{\partial x}\right)^{m}\right\}=-12 \mu \frac{\partial h}{\partial t} .
$$

The final shape of solution to the Reynolds equation (3.38) is formally given as follows 


$$
p(x, t)=p_{o}+\left[C^{(m)}\left(x_{o}, t\right)-C^{(m)}(x, t)\right]
$$

where

$$
\begin{aligned}
& C^{(m)}(x, t)=\int \frac{1}{F^{(m)}(x)}\left[12 \mu \int R\left(-\frac{\partial h}{\partial t}\right) d x\right]^{\frac{1}{m}} d x, \\
& F^{(m)}(x)=\left\{R\left[h^{m+2} \Phi^{(m)}(\chi)+12 \mu H \Phi_{p} \Psi^{(m)}(\Upsilon)\right]\right\}^{\frac{1}{m}} .
\end{aligned}
$$

In reality Eq.(3.8) is non-linear with respect to the pressure gradient $P\left(=-\frac{\partial p}{\partial x}\right)$ because it is also included into the functions $\Phi^{(m)}(\chi)$ and $\Psi^{(m)}(\Upsilon)$. One may propose the following simple iterative scheme to solve Eq.(3.8) with respect to $P$ using formulae (3.9) and (3.10)

$$
\chi_{(-1)}=0 \underset{E q .(3.8)}{\rightarrow} P_{(0)} \Rightarrow \chi_{(0)} \underset{E q .(3.8)}{\rightarrow} P_{(1)} \Rightarrow \chi_{(1)} \cdots \chi_{(k-1)} \underset{E q \cdot(3.8)}{\rightarrow} P_{(k)} .
$$

Using this scheme we will obtain

$$
p_{k}(x, t)=p_{o}+\left[C_{k}^{(m)}\left(x_{o}, t\right)-C_{k}^{(m)}(x, t)\right]
$$

where

$$
\begin{aligned}
& C_{k}^{(m)}(x, t)=\int \frac{1}{F_{k}^{(m)}(x)}\left[12 \mu \int R\left(-\frac{\partial h}{\partial t}\right) d x\right]^{\frac{1}{m}} d x, \\
& F_{k}^{(m)}(x)=\left\{R\left[h^{m+2} \Phi^{(m)}\left(\chi_{k-1}\right)+12 \mu H \Phi_{p} \Psi^{(m)}\left(\Upsilon_{k-1}\right)\right]\right\}^{\frac{1}{m}} .
\end{aligned}
$$

\section{Example of flow}

Let us consider the pressure distribution in a squeeze film bearing presented in Fig.2 lubricated with the Herschel-Bulkley fluid.

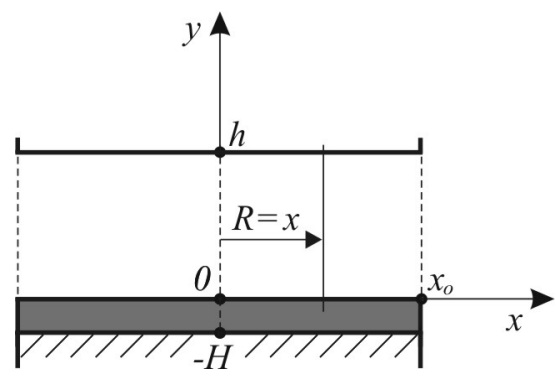

Fig.2. Step squeeze film bearing. 
Assume also that the lubricant flow coincides with a small core, then $\chi<<1, \Upsilon<<1$ and the functions $\Phi^{(m)}(\chi)$ and $\Psi^{(m)}(\Upsilon)$ become linear

$$
\begin{aligned}
& \Phi^{(m)}(\chi) \approx \frac{6}{2^{m}(m+2)}-\frac{6 m}{2^{m}(m+1)} \chi \\
& \Psi^{(m)}(\Upsilon)=\frac{1}{m+3}-\frac{m}{m+2} \Upsilon
\end{aligned}
$$

Using the iterative scheme (3.11) and introducing the following dimensionless variables and parameters

$$
\begin{aligned}
& \tilde{x}=\frac{x}{R_{o}}, \quad \tilde{R}=\frac{R}{R_{o}}, \quad \tilde{h}=\frac{h}{h_{o}}=\varepsilon, \quad K=\frac{r_{c}}{h_{o}}, \quad \tilde{H}=\frac{H \varphi_{p}}{h_{o}}, \quad h=h_{o} \varepsilon(t) \\
& \tilde{p}=\frac{\left(p-p_{o}\right) h_{o}^{\frac{2}{m}+1}}{\mu^{\frac{1}{m}} V_{o}^{\frac{1}{m}} R_{o}^{\frac{1}{m}+1}}, \quad \mathrm{SV}=\frac{\tau_{o} h_{o}^{\frac{2}{m}}}{\mu^{\frac{1}{m}} V_{o}^{\frac{1}{m}} R_{o}^{\frac{1}{m}}}, \quad V_{o}=h_{o}(-\dot{\varepsilon}), \quad \dot{\varepsilon}=\frac{d \varepsilon}{d t},
\end{aligned}
$$

one may present formula (3.12) for the pressure distribution in a step squeeze film bearing in a simple nondimensional form

$$
\tilde{p}(\tilde{x}, \tilde{t})=D^{(m)}\left(1-\tilde{x}^{\frac{1}{m}+1}\right)+E^{(m)}(1-\tilde{x}) .
$$

where

$$
D^{(m)}=\frac{2 m}{(m+1)\left(\frac{\varepsilon^{m+2}}{m+2}+\frac{2}{m+3} \tilde{H} K^{m+1}\right)^{\frac{1}{m}}}, \quad E^{(m)}=2 \mathrm{SV} \frac{\frac{\varepsilon^{m+1}}{m+1}+\frac{2 \tilde{H} K^{m}}{m+2}}{\frac{\varepsilon^{m+2}}{m+2}+\frac{2 \tilde{H} K^{m+1}}{m+3}}
$$

SV - is the Saint-Venant plasticity number (plasticity index).

The graphical presentation of the pressure distribution given by formula (4.3) is shown in Figs $3 \div 14$; the graphs presented in Figs 3, 4, 7, 8 and 11, 12 are made for $\varepsilon=1$ (the squeezing start) whereas the graphs presented in Figs 5, 6, 9, 10 and 13, 14 are made for $\varepsilon=0.5$. 


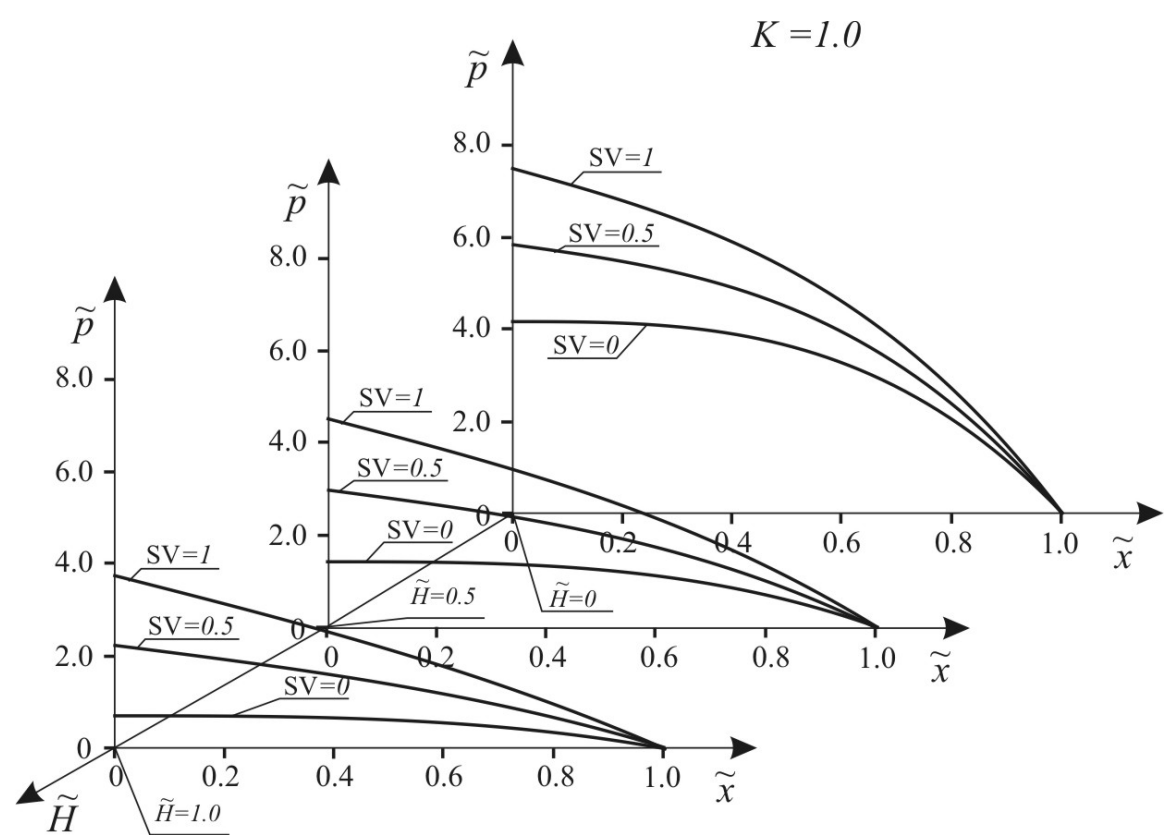

Fig.3. Dimensionless pressure distribution in the step bearing for $m=0.5, K=1.0$ and $\varepsilon=1.0$.

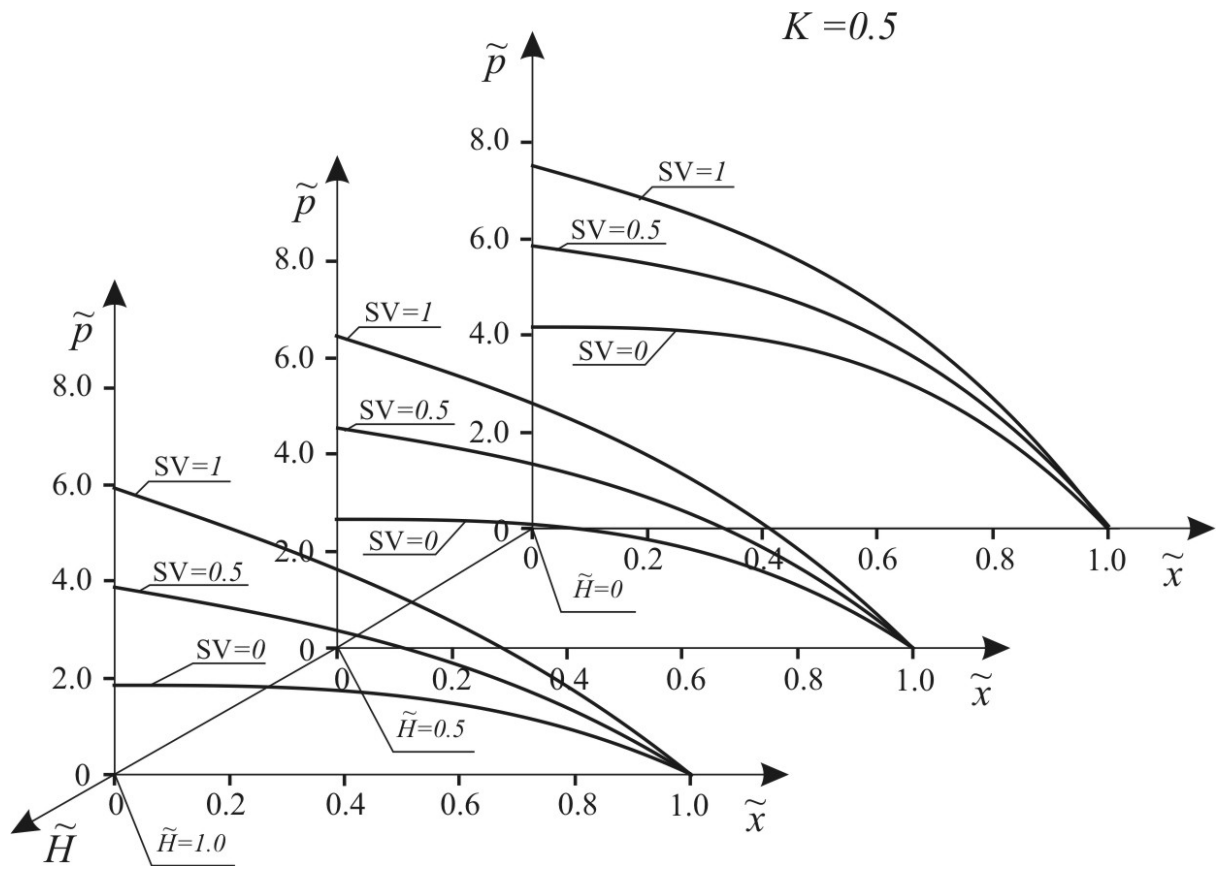

Fig.4. Dimensionless pressure distribution in the step bearing for $m=0.5, K=0.5$ and $\varepsilon=1.0$. 


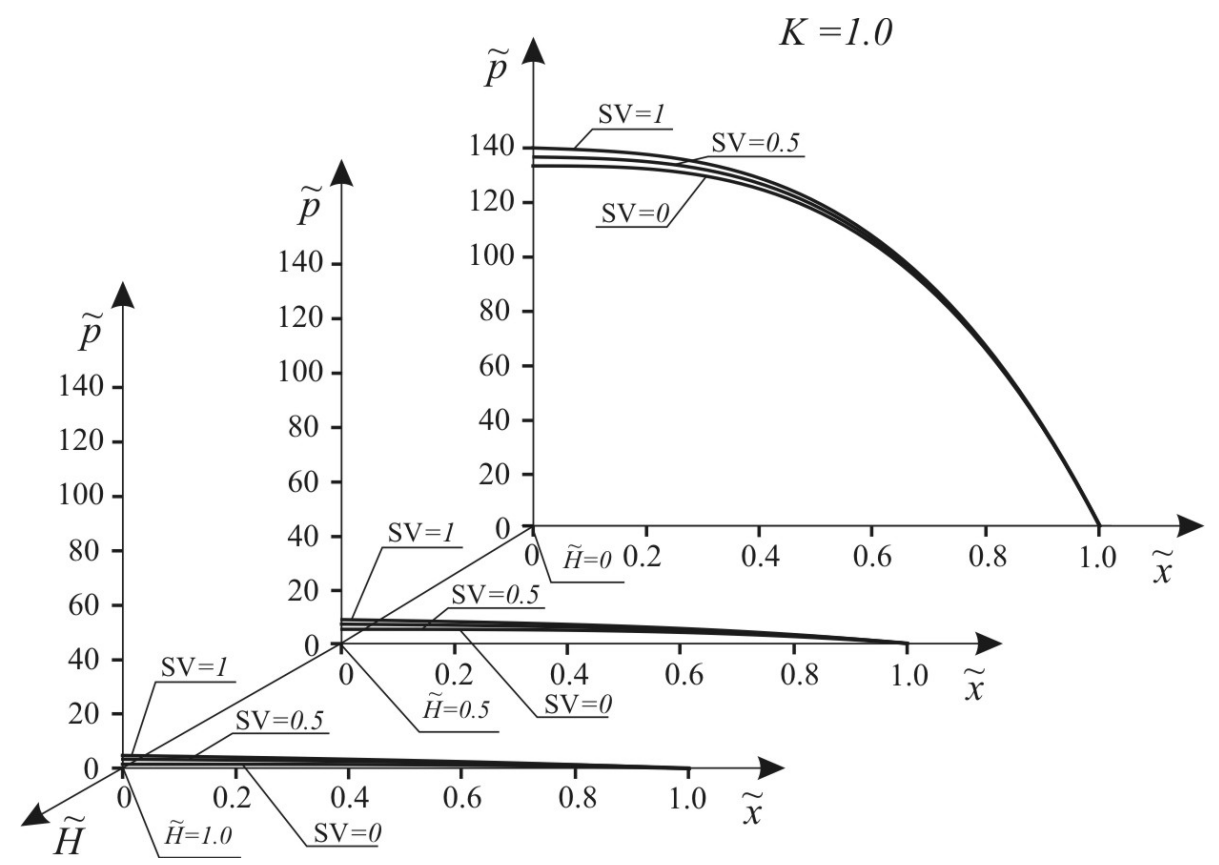

Fig.5. Dimensionless pressure distribution in the step bearing for $m=0.5, K=1.0$ and $\varepsilon=0.5$.

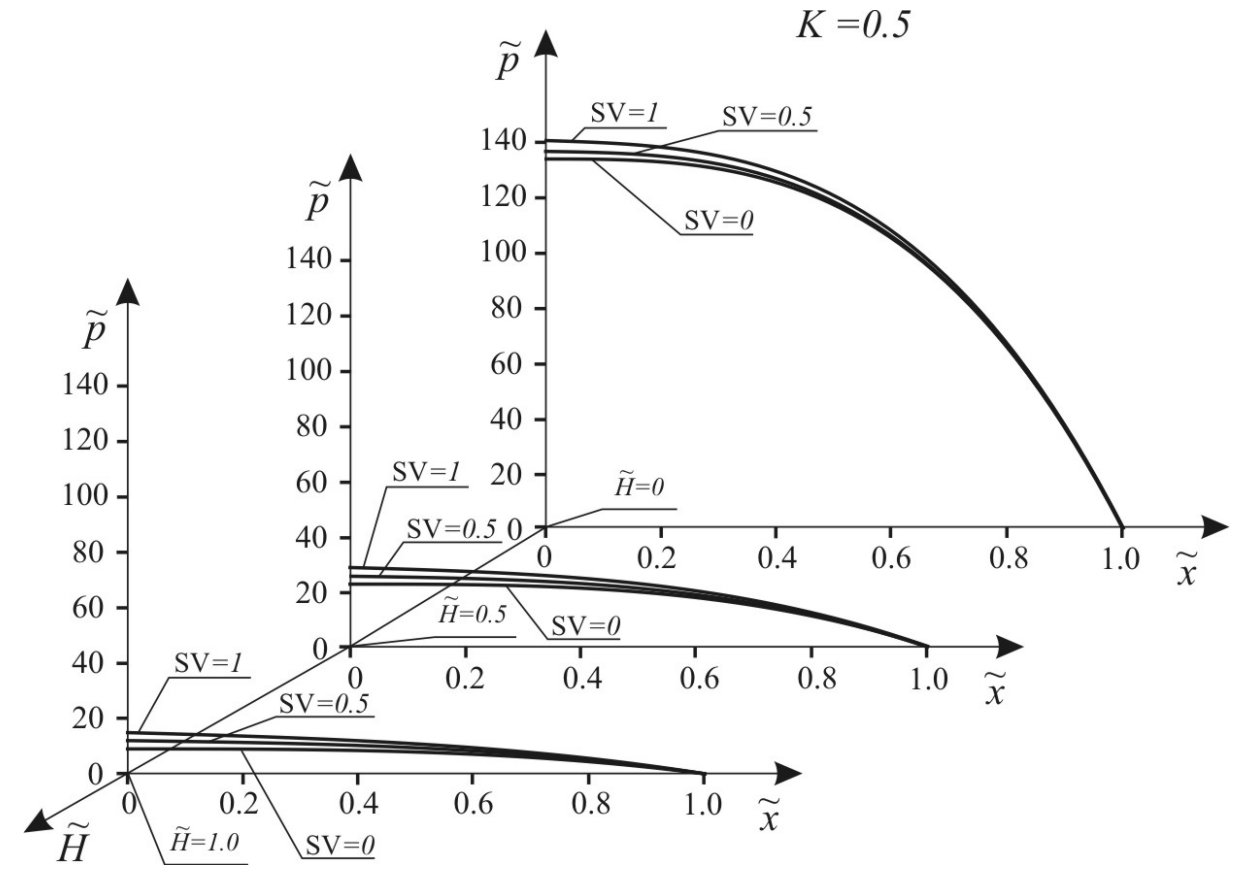

Fig.6. Dimensionless pressure distribution in the step bearing for $m=0.5, K=0.5$ and $\varepsilon=0.5$. 


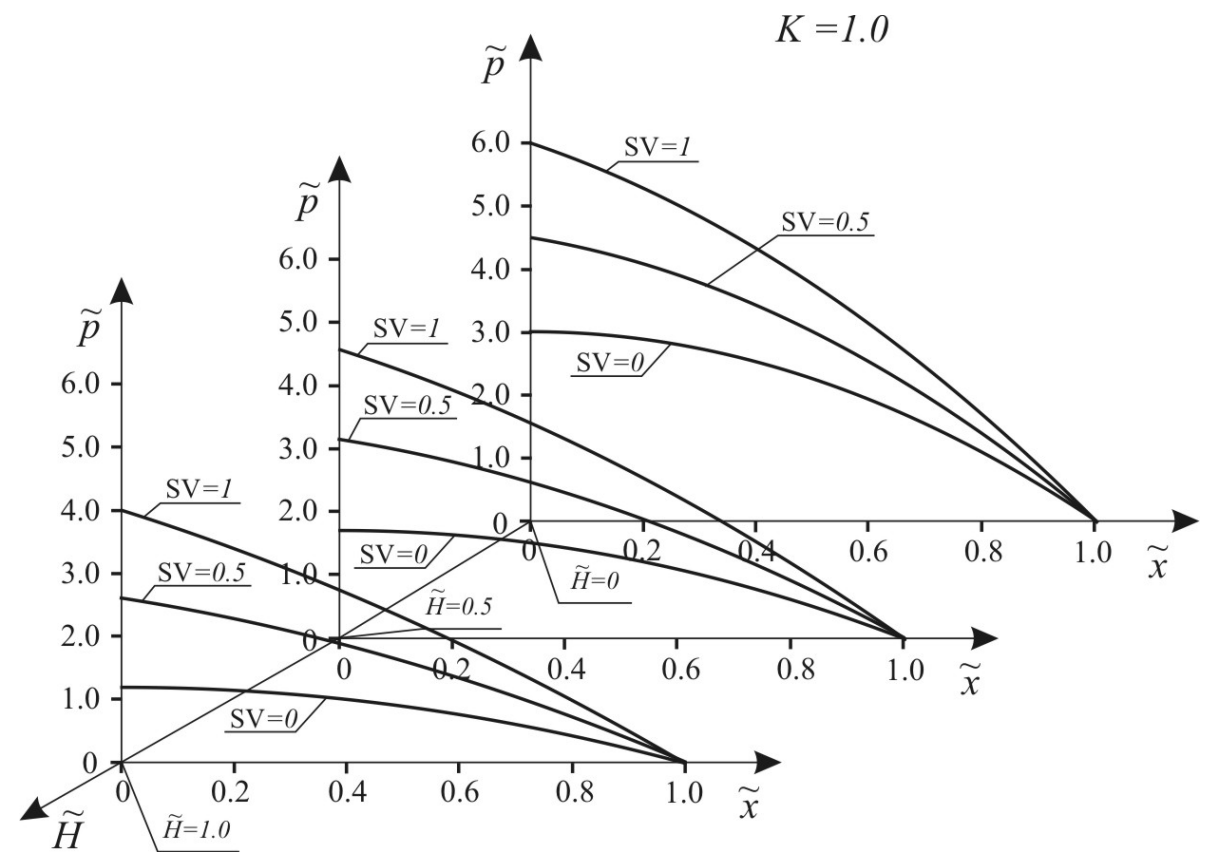

Fig.7. Dimensionless pressure distribution in the step bearing for $m=1.0, K=1.0$ and $\varepsilon=1.0$.

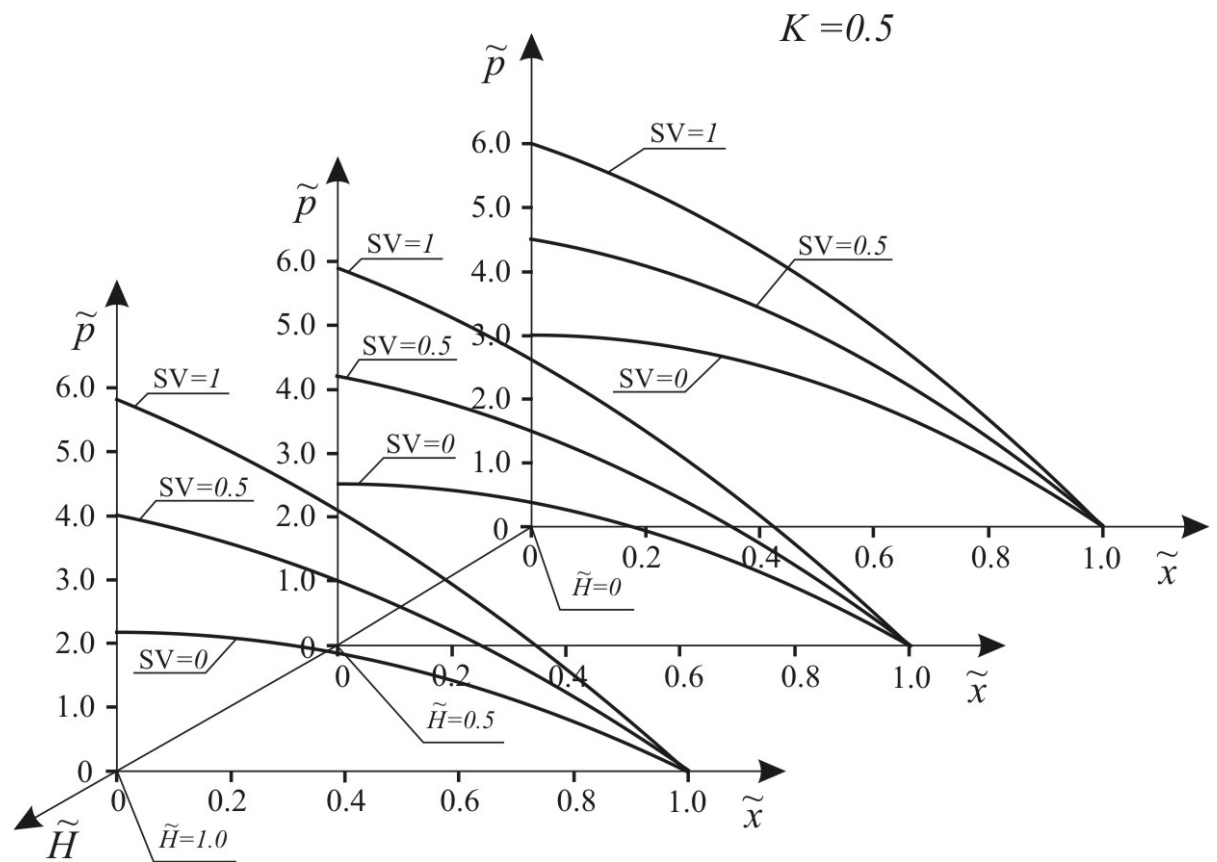

Fig.8. Dimensionless pressure distribution in the step bearing for $m=1.0, K=0.5$ and $\varepsilon=1.0$. 


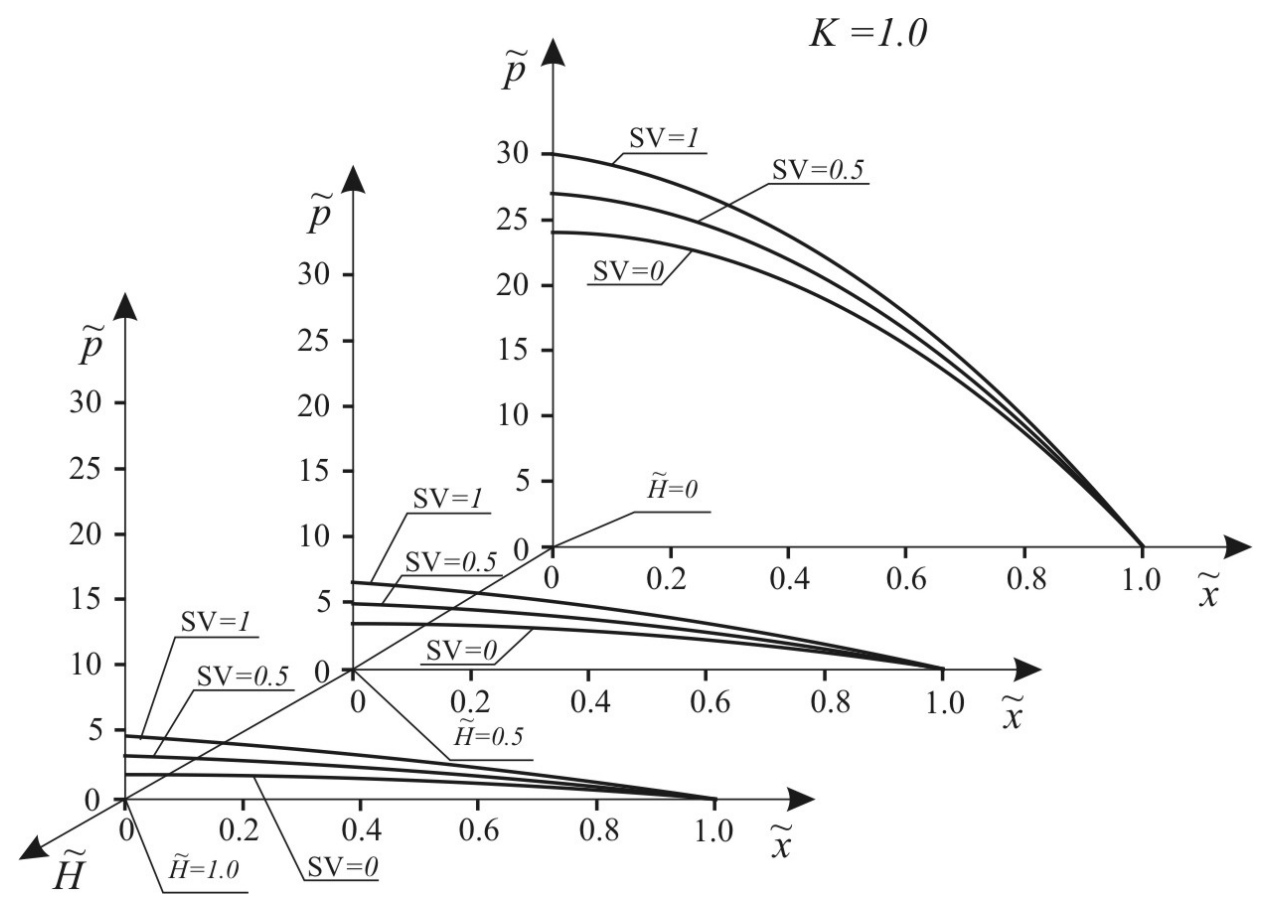

Fig.9. Dimensionless pressure distribution in the step bearing for $m=1.0, K=1.0$ and $\varepsilon=0.5$.

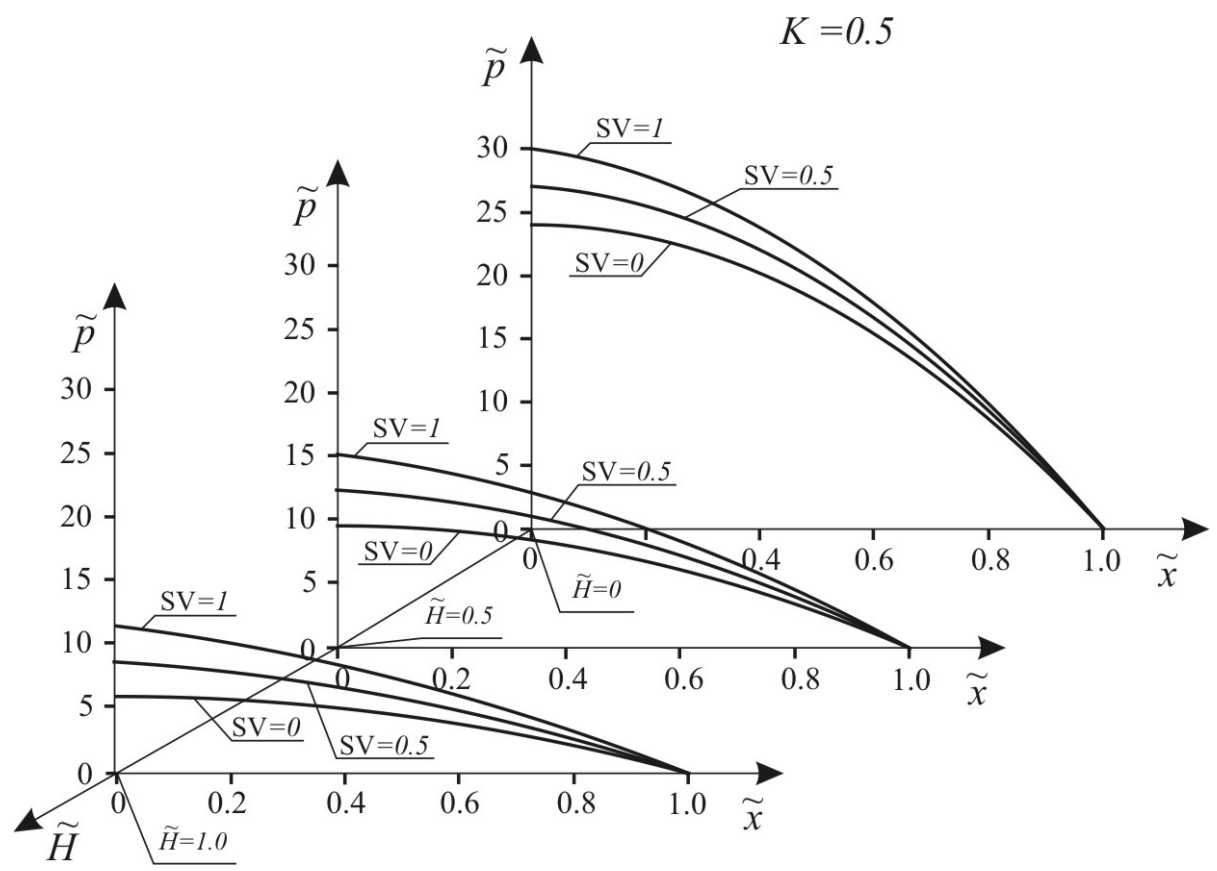

Fig.10. Dimensionless pressure distribution in the step bearing for $m=1.0, K=0.5$ and $\varepsilon=0.5$. 


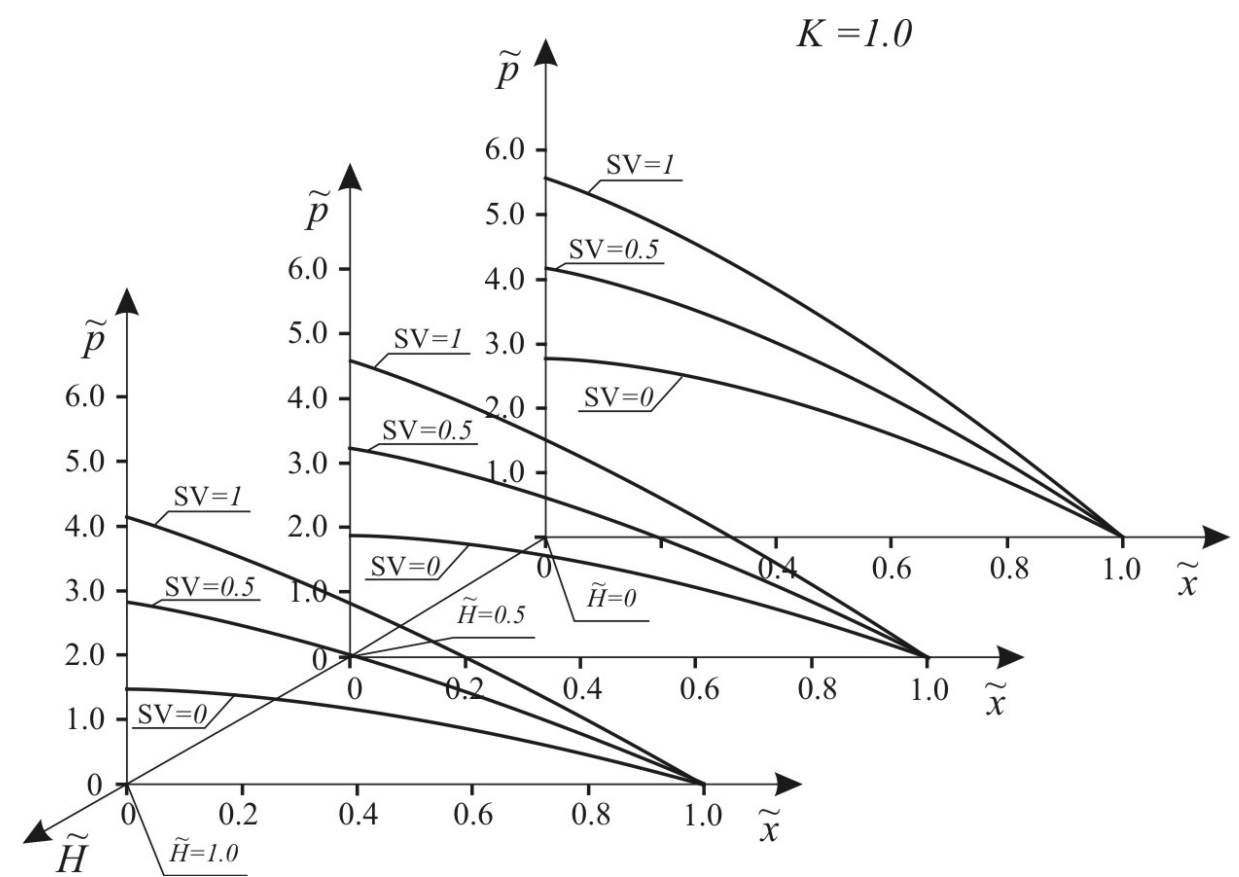

Fig.11. Dimensionless pressure distribution in the step bearing for $m=1.5, K=1.0$ and $\varepsilon=1.0$.

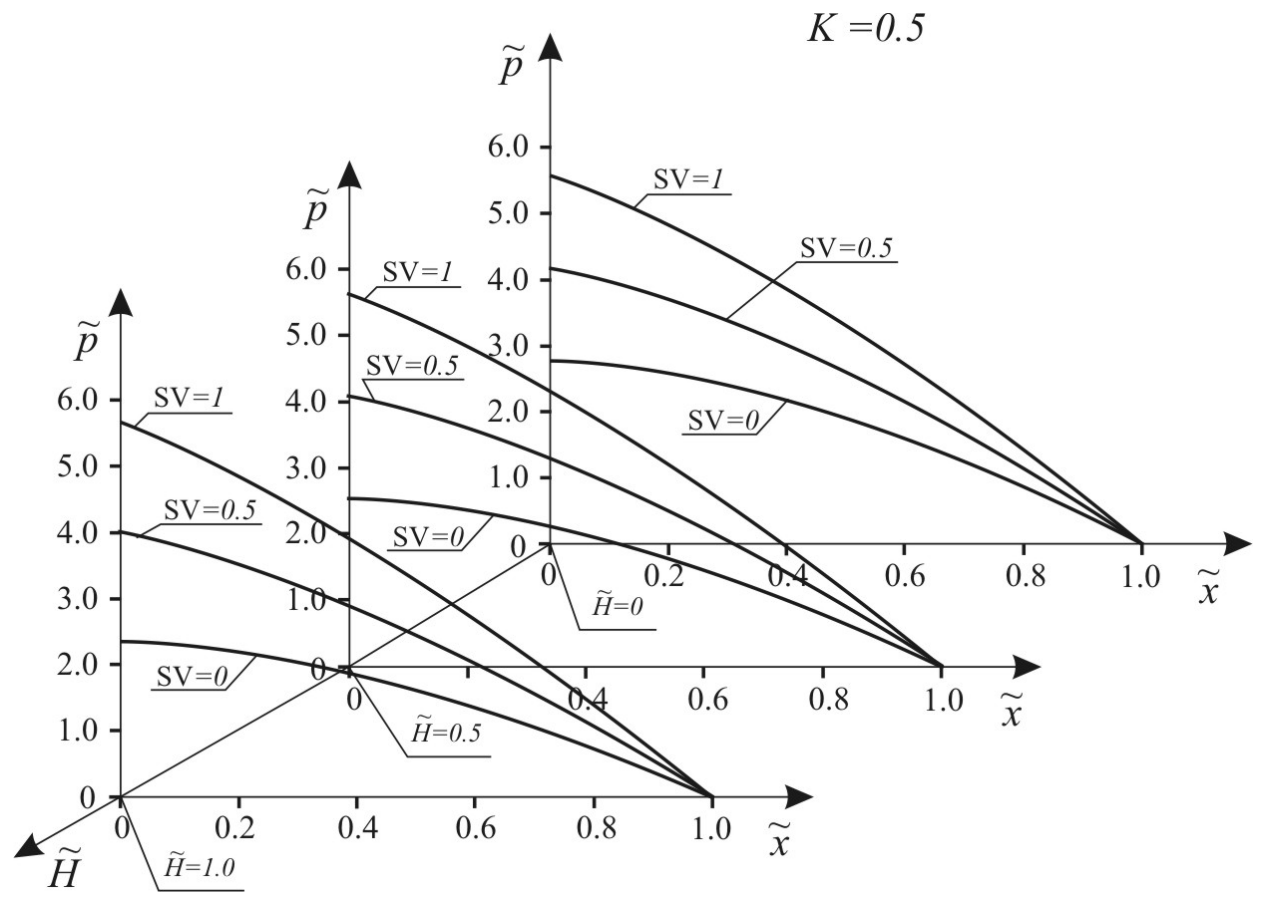

Fig.12. Dimensionless pressure distribution in the step bearing for $m=1.5, K=0.5$ and $\varepsilon=1.0$. 


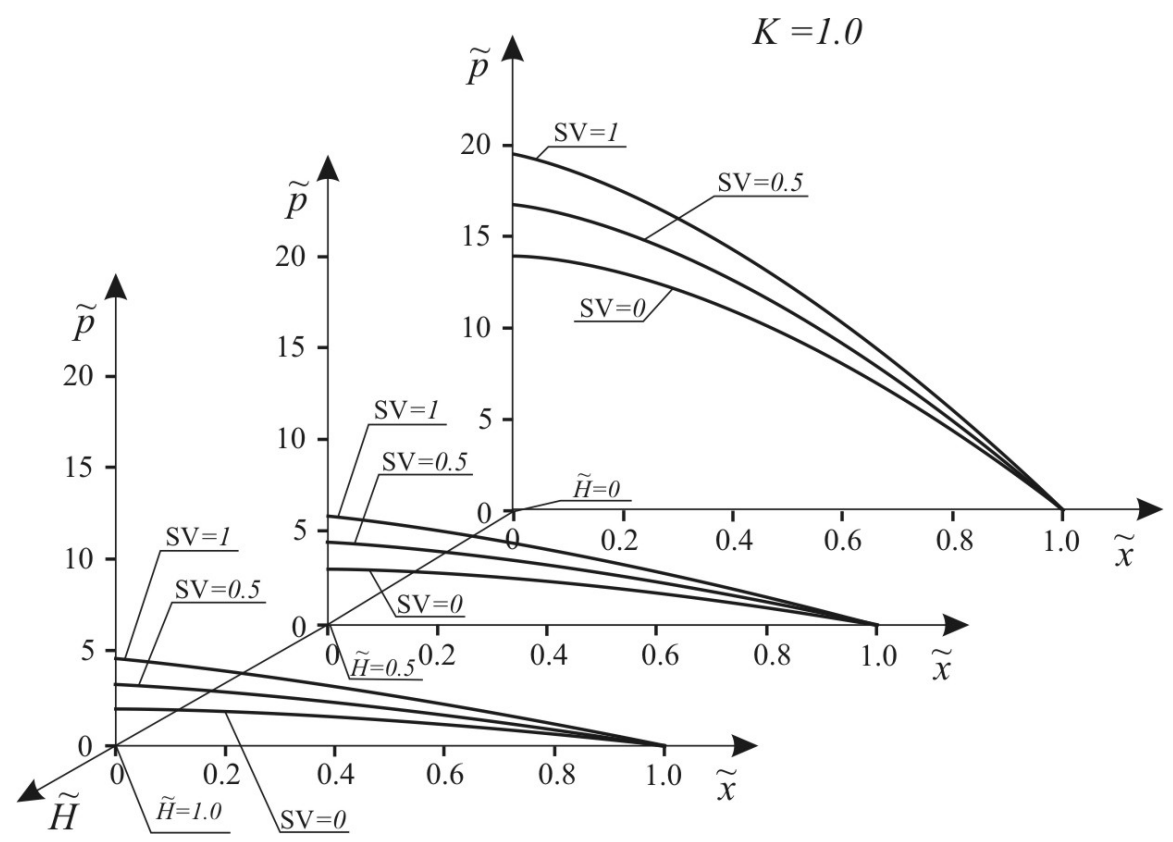

Fig.13. Dimensionless pressure distribution in the step bearing for $m=1.5, K=1.0$ and $\varepsilon=0.5$.

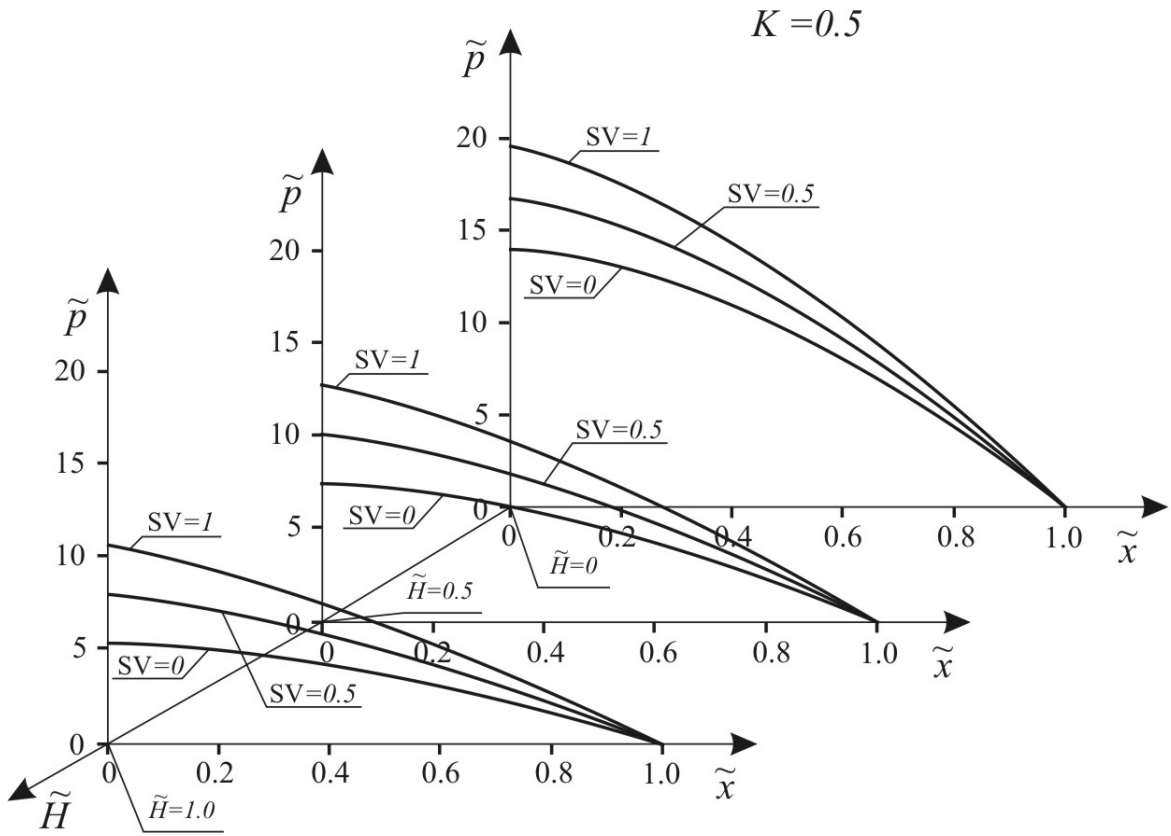

Fig.14. Dimensionless pressure distribution in the step bearing for $m=1.5, K=0.5$ and $\varepsilon=0.5$.

\section{Discussion and conclusion}

The above analysis has shown that Reynolds approximation may be used to model the flow of the Hershel-Bulkley fluid as the lubricant in the clearance of a curvilinear squeeze film bearing.

The obtained results are relatively simple and may be used in practical applications. 
For a step bearing the results have been presented for three values of the Herschel-Bulkley exponent, namely: for $m=0.5, m=1.0$ (Bingham fluid) and for $m=1.5$ and also for different parameters of the porous matrix (different values of $\tilde{H}$ and $K$ ).

For $m=0.5$ when squeezing starts the values of the pressure are not large and generally they decrease with the increase of the values of $K$ and $\tilde{H}$.

In the full squeezing the pressures are very great and they decrease very quickly with the increase of the values of $K$ and $\tilde{H}$.

For $m=1.0$ the values of the pressure are large at squeezing and they are lager in the full squeezing; they decrease with the increase of the values of $K$ and $\tilde{H}$.

For $m=1.5$ all values of the pressure decrease with the increase of the values of $K$ and $\tilde{H}$.

It may be concluded that for a squeeze film bearing lubricated with a lubricant of the HerschelBulkley type it is more profitable to take the lubricant with $m<1.0$.

\section{Nomenclature}

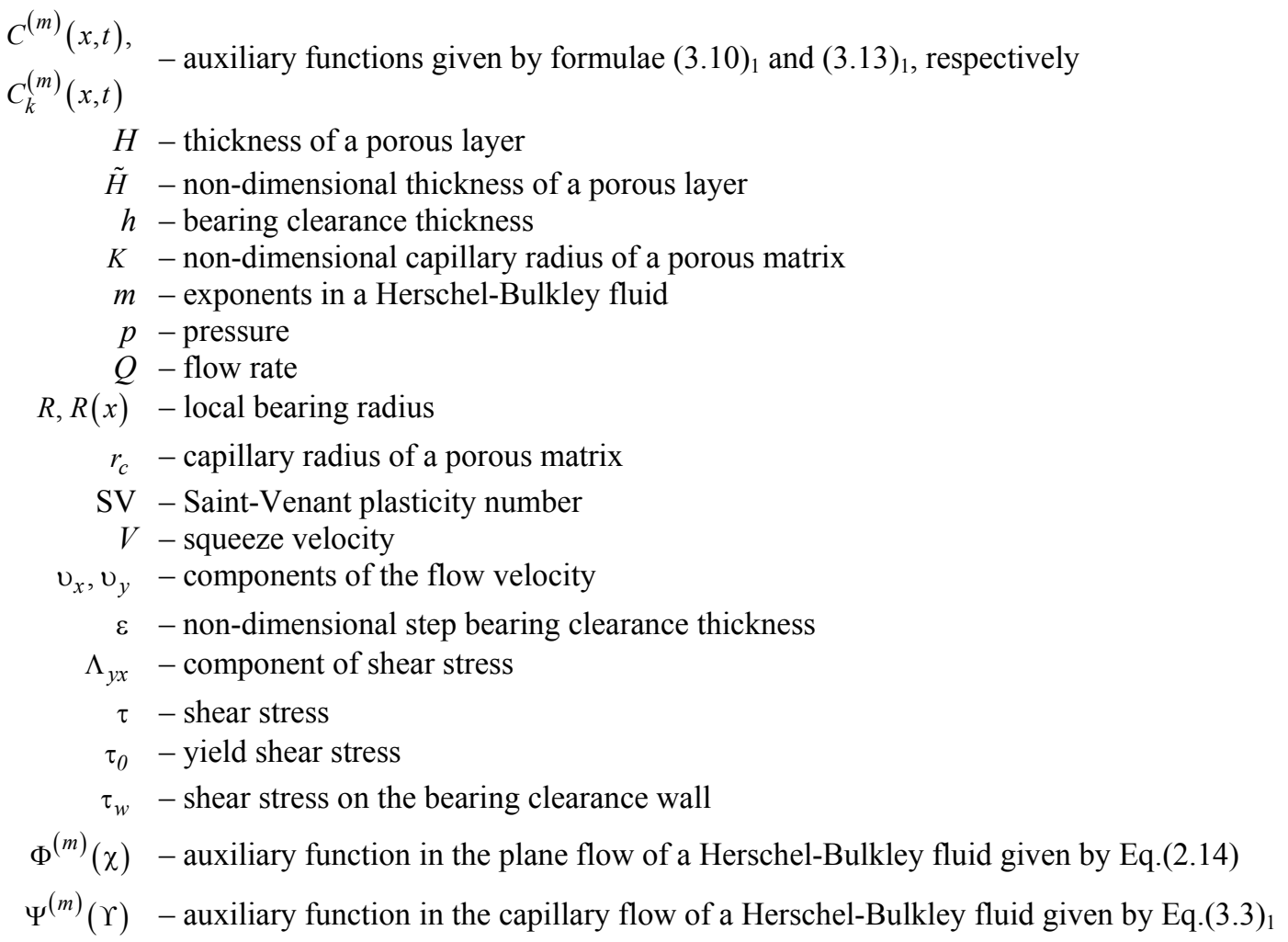

\section{References}

[1] Adams M.J. and Edmondson B. (1987): Forces between particles in continuous and discrete liquid media. - In: B.J.Briscoe and M.J.Adams (Eds) IOP Publishing, New York: Tribology in particulate technology, pp.154-172.

[2] Covey G.H. and Stanmore B.R. (1981): Use of the parallel-plate plastometer for the characterisation of viscous fluids with a yield stress. - J. Non-Newtonian Fluid Mech., vol.8, pp.249-260.

[3] Dai G. and Bird R.B. (1981): Radial flow of Bingham fluid between two fixed circular disks. - J. Non-Newtonian Fluid Mech., vol.8, pp.349-355.

[4] Lipscomb C.C. and Denn M.M. (1984): Flow of Bingham fluids in complex geometries. - J. Non-Newtonian Fluid Mech., vol.14, pp.337-349. 
[5] Rodin G.J. (1996): Squeeze film between two spheres in a power-law fluid. - J. Non-Newtonian Fluid Mech., vol.63, pp.141-152.

[6] Smyrnaios D.N. and Tsamopoulos J.A. (2001): Squeeze flow of Bingham plastic. - J. Non-Newtonian Fluid Mech., vol.100, pp.165-190.

[7] Vishwanath K.P and Kandasamy A. (2010): Inertia effects in circular squeeze film bearing using Herschel-Bulkley lubricants. - Appl. Math. Modelling, vol.34, pp.219-227.

[8] Engman J., Servais C. and Burbidge A.S. (2005): Squeeze flow theory and applications to rheometry: a review. J. Non-Newtonian Fluid Mech., vol.132, pp.1-27.

[9] Xu C., Yuan L., Xu Y. and Hang W. (2010): Squeeze flow of interstitial Herschel-Bulkley fluid between two rigid spheres. - Particuology, vol.8, pp.360-364.

[10] Walicka A. (1994): Accurate and Asymptotic Solutions of Viscous Fluids in a Clearance Bounded by Two Coaxial Surfaces of Revolution (in Polish). - Warsaw: WN-T.

[11] Bujurke N.M., Jagadee M. and Hiremath P.S. (1987): Analysis of normal stress effects in squeeze film porous bearing. - Wear, vol.116, pp.237-248.

[12] Etsion I. and Michael O. (1994): Enhancing sealing and dynamic performance with partially porous mechanical face seals. - Trib. Transactions, vol.37, pp.701-710.

[13] Morgan V.T. and Cameron A. (1957): Mechanism of lubrication in porous metal bearings. - In: Proc. Conf. on Lubrication and Wear, Inst. Mech. Eng. London, pp.151-157.

[14] Prakash J. and Vij S.K. (1973): Load capacity and time-height relations for squeeze films between porous plates. Wear, vol.24, pp.309-322.

[15] Shukla J.B. and Isa M. (1978): Externally pressurised porous thrust bearing with power-law lubricant. - Wear, vol.33, pp.85-92.

[16] Walicki E, Walicka A. and Makhaniok A. (2000): Pressure distribution in a curvilinear thrust bearing with one porous wall lubricated by a Bingham fluid. - In: Proc. First 2000 AIMETA Int. Trib. Conf., L'Aquila, Italy 2000, pp.528-539.

[17] Walicka A. (2011): Pressure distribution in a porous curvilinear squeeze film bearing lubricated by a Bingham fluid. - Int. J. Appl. Mech. Enging, vol.16, pp.1215-1224.

[18] Falicki J. (2007): The Influence of Viscoplastic Lubricants on the Pressure Distributions in Thrust Slide Bearings (in Polish). - PhD Thesis, University of Zielona Gora, p.221.

[19] Walicki E. (2005): Rheodynamics of the Lubrication of Slide Bearings (in Polish). - Zielona Góra: University Press, p.556.

[20] Herschel W.M. (1922): Viscosity and friction. - SAE Journal, vol.10, pp.31-38.

[21] Herschel W.M. and Bulkley R. (1926): Konsistenzmessungen von Gummi-Benzollosungen. - Kolloid Z., vol.39, pp.291297.

[22] Walicki E. and Walicka A. (1997): Throughflow of viscoplastic fluids between fixed surface of revolution. - In: Proc. $5^{\text {th }}$ Nat. Conf. on Multiphase Flows 1997, Gdańsk, Poland, vol.2, pp.133-136.

[23] Walicka A. (2002): Rheodynamics of Non-Newtonian Fluids Flows in Straight and Curved Channels (in Polish). Zielona Góra: University Press, p.307.

[24] Walicka A. (2002): Rotational Flows of the Rheologically Complex Media in Thin Annular Channels (in Russian). - Zielona Góra: University Press, p.385.

[25] Walicka A., Jurczak P. and Walicki E. (2011): Generalized second grade fluids - Basic equations and basic flows. - In EKMA Press, Warsaw: Rheology - Theory and Application, vol.2, pp.303-335.

[26] Walicka A. and Walicki E. (2011): Non-Newtonian fluids flows in porous media. - In EKMA Press, Warsaw: Rheology - Theory and Application, vol.2, pp.337-367. 